\title{
A Resection Method Applied To Infrared Measurements
}

\section{Y. Le Sant*}

*Office National d'Etudes et de Recherches Aérospatiales, Chatillon, France

\begin{abstract}
Heat flux measurements are one of the main purposes of tests carried out in hypersonic wind tunnels. They are performed at Onera mainly with Infrared Thermography (IRT), while sensors as thermocouples enable to check its reliability. Image processing tools are used to recognize the model position in images and to extract information from them. Methods doing these actions are called resection methods. The Onera's method requires markers, which are recognized automatically. The relative position of the camera is identified and the model motion can then be corrected in a 3D manner. The resection method is now widely applied for wind tunnel testing both for infrared or visible applications.
\end{abstract}

\section{Introduction}
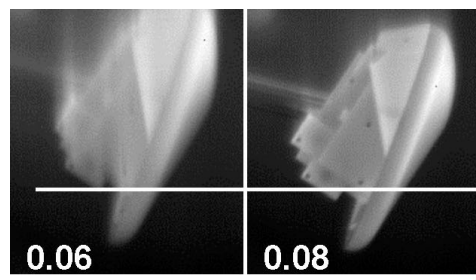
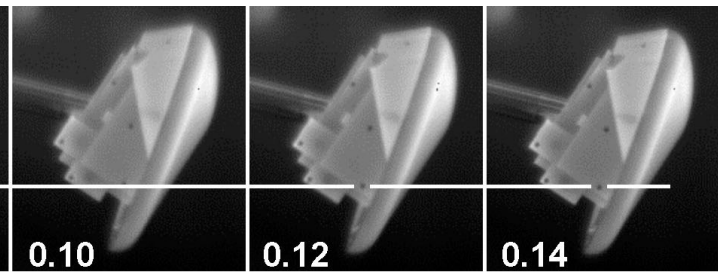

Fig.1. Model injection sequence.

Onera uses IRT for heat flux measurements in hypersonic wind tunnels. The model can be injected in the flow, thus creating a model motion. Figure 1 shows an injection sequence in the Onera's hypersonic wind tunnel S4 located at Modane, France. The first image cannot be used because it is too blurred. Blurring is acceptable or negligible in the other images, but a model motion is visible as it is highlighted with the white line. Model vibration or displacement due to aerodynamic loading can also induce a model motion as in figure 2 : displacements are quite small but they must be taken into account.

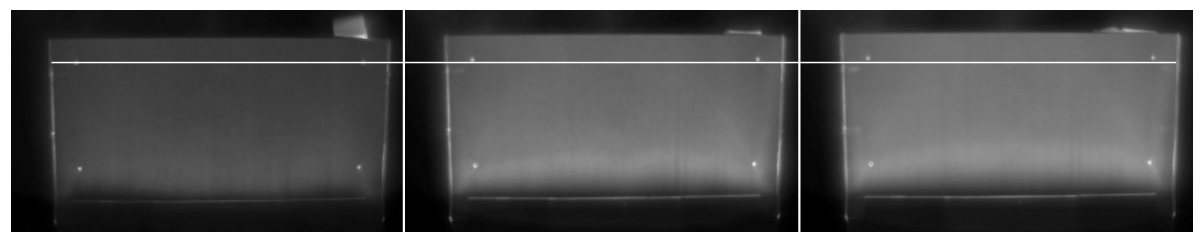

Fig.2. Model motion due to aerodynamic loading.

Since the whole temperature history is required for a proper heat flux data reduction, the model motion must be corrected for all images. This implies to recognize the model location in each image, which is the very first objective of the Onera's resection software AFIX2 [1,2] (freeware available at www.onera.fr/dafe- 
en/afix2) devoted to optical quantitative mapping techniques applied in wind tunnel testing, see section §2. AFIX2 links the model in the image with the real model so that every pixel is connected with a real point on the model, see section $\S 3$. This enables to compensate the model motion and to extract information at the wanted locations. Section $\S 4$ shows that IRT benefits of some interesting features of the method, as to apply emissivity correction. Section $\$ 5$ described an advanced model motion correction method implemented in AFIX2.

\section{Finding the model in the image}

\subsection{What is a resection method}

Linking the image to the real world for wind tunnel applications started to be addressed in the late 80's. The first developments were made for IRT and TSP (Thermal Sensitive Paint) applications. However, the development of the Pressure Sensitive Paint measurement technique (PSP) $[3,4]$ was the main motivation for developing efficient image processing softwares [5]. PSP requires extremely accurate image alignment and this is why the word resection has been introduced because it is a kind of image surgery. A resection method enables to identify the model in the image, to align images accurately and to link every pixel to a point on the model. In addition, the method is used in a productive environment so it must be fast and nearly fully automatic. This chapter describes the main features of the Onera resection software AFIX2 for IRT applications.

\subsection{Markers}

The black disks in figure 1 are markers created with aluminum stickers. They are visible even at room temperature because of their low emissivity. The white markers in figure 2 were created with plaster : their surface temperature is higher than the surrounding model temperature because of the plaster insulating properties. A marker detection tool called SPOT in AFIX2 enables to detect markers having a given diameter. It performes a correlation with a top hat pattern. A threshold is applied on the correlation value and the marker center is obtained by applying an average weighted with the correlation value. The uncertainty is lower than 0.1 pixel, which is enough for IRT applications. Figure 3a shows the result of the detection on images in figure 1. All the markers have been recognized as well as false markers which are edges or model defects.
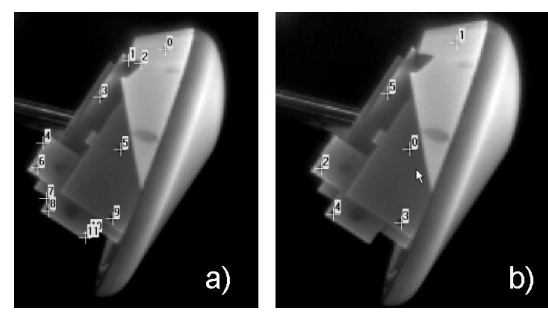

Fig. 3. a) Marker detection with SPOT b) Matched markers.

A matching tool is used to select the real markers. It requires a preliminary selection of the true markers in a reference image. Three markers among them are selected which are expected to be viewed in all images. Then for each proceeded image, they are randomly connected to image markers. A polynomial transformation 
between the image and the reference image is established. It is applied on all the markers and the matched markers are counted. The right connection in figure $3 \mathrm{~b}$ is the one that connects the greatest marker number.

\subsection{Determining the Point of View (PoV) of the camera}

Matched markers are known by their image coordinates and their space coordinates. They are used to identify the Point of View (PoV) of the camera. It is defined by six parameters which are three rotations and three translations, see figure 4. These parameters are gathered in a matrix system that transforms the space coordinates in camera coordinates. The pinhole model, see figure 5 , is used to transform the camera coordinates in image coordinates. Its parameters are the image size and the distance of the principal point $C$ to the image plane, which is related to the focal distance of the real lens.

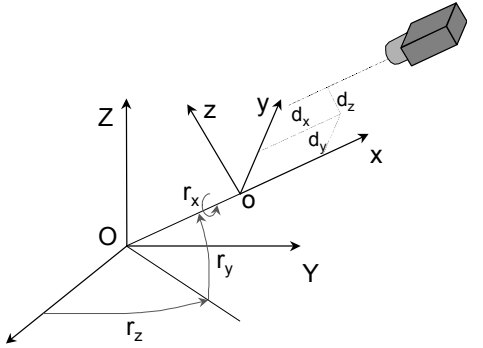

Fig. 4. PoV definition.

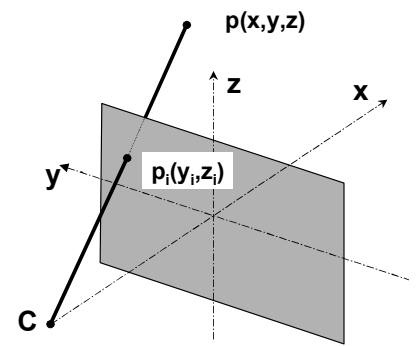

Fig. 5. Lens model.

The only one drawback of the matrix system is that it cannot be inverted easily because the rotations are mixed in the rotation matrix with cosine and sine functions. As a result, photogrammetry equations Eqs. (1) are often used because they can be inverted. They are equivalent to the original matrix system, but they involve eleven coefficients while the matrix system depends only on six parameters. As a consequence, six markers are required to identify the PoV using the photogrammetry equations instead of three markers with the matrix system. This is the reason why AFIX2 keeps using the original matrix system.

$$
y_{i}=\frac{a_{y x} X+a_{y y} Y+a_{y z} Z+a_{y}}{b_{x} X+b_{y} Y+b_{z} Z+1} \quad z_{i}=\frac{a_{z x} X+a_{z y} Y+a_{z z} Z+a_{z}}{b_{x} X+b_{y} Y+b_{z} Z+1}
$$

The PoV identification algorithm is a RMS method which minimizes the distance between the image marker coordinates and the coordinates computed according to the PoV. It is an iterative method and its original feature is that it can modify the parameter steps at each iteration. Each parameter cannot be modified by more than three steps at each iteration, which ensures that no parameter is going to be optimized while the others are far from the solution. The algorithm is fast, robust and requires only an initial rough guess of the PoV.

A grid is required to support the PoV identification, as in figure 6. It is built with triangles. The reliability of the PoV can be checked by superimposing the model contour, at is shown in figure 7. The RMS value is usually lower than 1 pixel. 


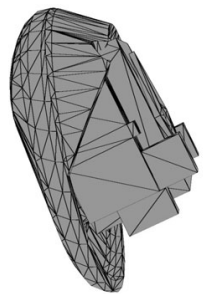

Fig. 6. Grid of the model.

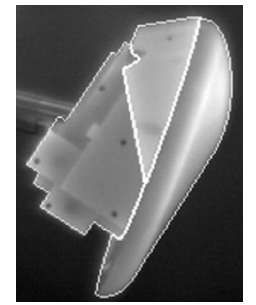

Fig.7. Grid contour superimposed.

For some applications it is not possible to use markers or they are not required because the model contour provides a lot of information. It is then possible to adjust manually the six PoV parameters. Dedicated tools in AFIX2 enable to handle PoV parameters and to see their effect on the grid wrapping. Figure 8 is a row image and figure 9 is the same image with the grid contour superimposed.

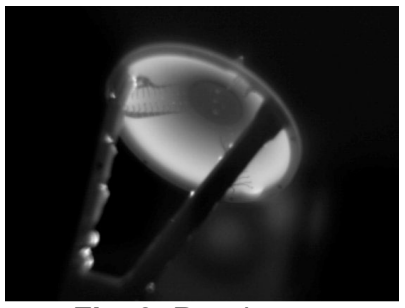

Fig. 8. Row image.

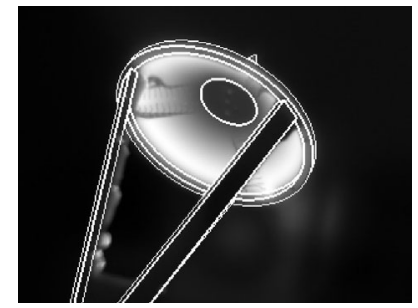

Fig.9. Grid contour superimposed.

\section{3D mapping}

When the PoV has been identified, each point on the model can be related to a pixel in the image. What is more useful is to relate each pixel to a point on the model. This is done by projecting the vertices of the grid triangles on the image. Then a bilinear interpolation is done on the projected triangle to relate each pixel with a point on the model. By doing this, each pixel is known by its image coordinates and spatial coordinates.
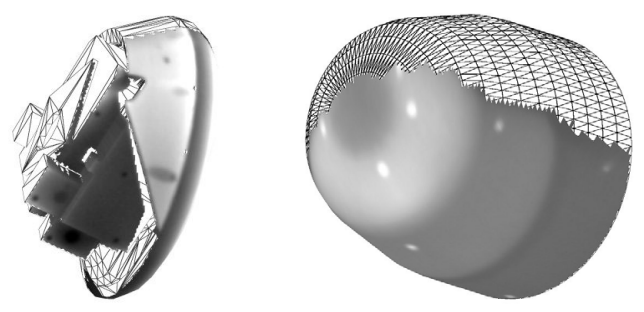

Fig. 10. Virtual images

Virtual images can be computed according to a virtual PoV. The spatial coordinates of the pixel are used to compute the image coordinates in the virtual image. This enables to compensate the model motion : all images are recomputed according to an unique PoV. Virtual images are used to combine several views or to help for flow understanding. They are often used to create views according to a perfect $P o V$ as a top view. Figure 10 shows typical virtual images where the grid is superimposed on areas not viewed in the real image. 
3D mapping enables to extract data from images. If the results are required on a given point set or on a line, the image coordinates of the points are calculated and used to get the wanted information. Figure 11 is an example where a comparison is made on lines on an axisymmetric model.
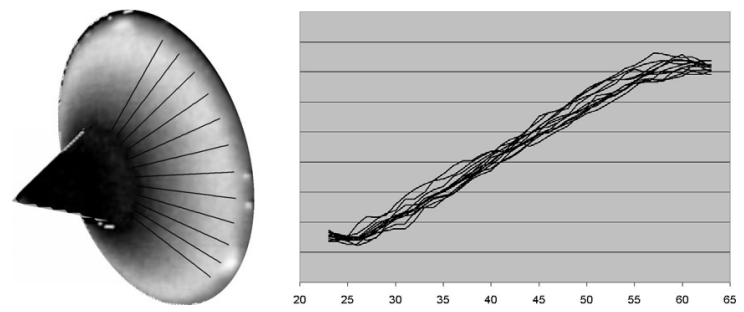

Fig. 11. Data extraction.

\section{Resection and IRT data reduction}

IRT data reduction benefits from the image recognition results. For example, the viewing angle is known for each pixel which enables to apply an emissivity correction. Figure 12 shows the image angle of the model presented in figure 11 . One other useful possibility is to compute image masks used to modify some parameters of the data reduction, as the thickness of the model or its thermal properties.

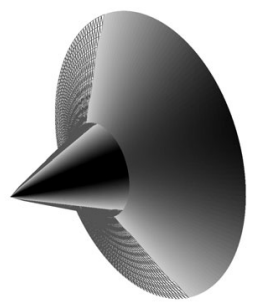

Fig. 12. Viewing angle map.

\section{An advanced model motion correction method}

The presented resection method enables to compensate the model motion. However, it works by using the whole set of markers and problem may arise at the beginning of the run because some of them may be not visible. An advanced solution is available which has mainly been developed to assess model deformation in the transonic range [6]. This solution can be used to model the motion which depends on a few parameters : usually a single translation is enough. The motion model is used to modify the spatial location of the grid. The advantage of this method is that only one marker may be enough. Moreover, a pure translation can be assessed with only one marker coordinate : not matter if the second coordinate is correct or not.

The method works by using a real or a virtual marker in the image. For example, images in figure 2 are enhanced at the bottom of the image. A bright area can then be seen where an insulating material was installed. A virtual marker on this line can be selected as in figure 13 . This can be done automatically when a real 
marker is used or manually with a virtual marker. A macro system working with AFIX2 is used to proceed the whole IR film.
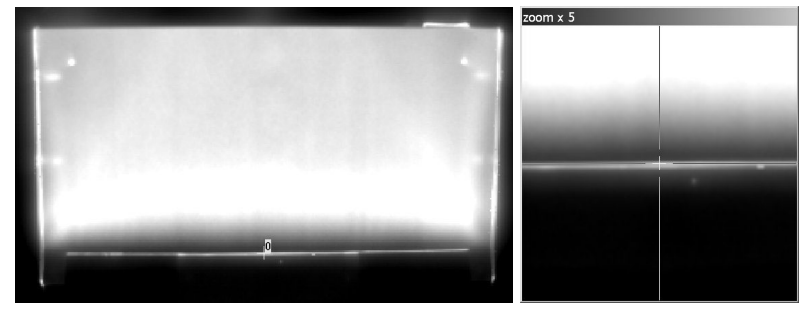

Fig. 13. Model motion assessment with a virtual marker.

\section{Concluding remarks}

The resection method developed mainly for PSP applications is now applied for IRT applications. It enables a nearly fully automatic data reduction, even when a model motion occurs. The method requires markers otherwise images must be proceeded manually. In such case, tools implemented in AFIX2 help to minimize the user effort so that a just a few minutes are needed to proceed an IRT film where there is a model motion.

\section{REFERENCES}

[1] Le Sant, Y.; Marchand, M.; Millan P. and, Fontaine F., An overview of infrared thermography techniques used in large wind tunnels. Aerospace Science and Technology, Vol 6, Issue 5. Pages 355-366, September 2002.

[2] Le Sant, Y.; Deléglise , B. and Mébarki, Y. An automatic image alignment method applied to pressure sensitive paint measurements.17th International Congress on Instrumentation in Aerospace Simulation Facilities, Monterey (U.S.A.), September 29, October 1997.

[3] Bell J.H.; Shairer E.T.; Hand L.A. and Mehta R.D. Surface Pressure Measurements Using Luminescent Coatings, Annual Review of Fluid Mechanics, vol. 33, pp. 155-206, March 2001.

[4] Sullivan, J. Temperature and Pressure Sensitive Paint, Lecture Series 20002001, Advanced Measurement Techniques, Von Karman Institute for Fluid Mechanics, January 2001.

[5] Bell, J.H. and McLachlan, B.G. Image Registration for Luminescent Paint Sensors. $31^{\text {st }}$ AIAA Aerospace Sciences Meeting and Exhibit, Paper AIAA 930178, Reno, NV, January 1993.

[6] Le Sant, Y. A Model Deformation Method Applied to PSP Measurements, International Congress on Instrumentation in Aerospace Simulation Facilities, ICIASF'03, 24-28 August 2003,Göttingen, Germany. 